\title{
Observing a p-n Junction in a Reverse-Biased GaP Light-Emitting Diode by Combining Electron Holography and Focused-Ion-Beam Milling
}

\author{
Z. Wang*, K. Sasaki**, T. Hirayama*, Y. Yabuuchi***, H. Saka** \\ * Japan Fine Ceramics Center (JFCC), Nagoya, 456-8587 Japan \\ ** Department of Quantum Engineering, Nagoya University, Nagoya, 464-8603 Japan \\ *** Matsushita Techno-research, Inc., Osaka, 570-8501 Japan
}

Electron holographic technique based on a transmission electron microscope (TEM) has been used to map and characterize two-dimensional electrostatic potential distributions across $p-n$ junctions in silicon devices with high resolution $[1,2]$. In particular, this technique, has been recently, used to successfully observe a $\mathrm{p}-\mathrm{n}$ junction under a reverse-bias condition in a $\mathrm{Si} / \mathrm{Si} \mathrm{p}-\mathrm{n}$ junction film prepared by $30-\mathrm{kV}$ gallium focused-ion-beam (FIB) milling [3]. The current report presents an in-situ observation of the potential distribution of a $p-n$ junction in a reverse-biased GaP lightemitting diode (LED) by combination of electron holography and FIB milling.

To apply a voltage externally to the GaP LED sample, a special sample stage, which enables the sample to be easily fixed and bonded to a holder with two electrodes, was developed [4]. The holder can be compatible with both Hitachi's FIB system and HF-2000 TEM equipped with an electron biprism. Figure 1 shows a scanning ion microscopic (SIM) image of the bonded GaP LED sample in the FIB system. To find the exact location of the p-n junction, the sample was examined firstly by electron holography under the reverse-bias condition. Figure 2(a) shows the electron hologram taken in vacuum near the p-n junction. Figure 2(b) is the reconstructed interference image, in which the external electrostatic-field distribution can be seen clearly around the junction. The sample was then placed in the FIB system, and an electron-transparent membrane was fabricated carefully at the junction position by using $30-\mathrm{kV}$ gallium FIB. However, it was not possible to observe the apparent potential change across the junction in the film even under reverse bias. This is because FIB milling introduced a damage layer with low resistivity on the film surface, which leaked the electric current over the junction during applying reverse bias. To reduce the damaged layer, the surface of the film was therefore milled for about one minute by an argon ion beam at an accelerating voltage of $7 \mathrm{kV}$. The final sample was examined by electron holography at different reverse-bias voltages. Figure 3 shows the reconstructed phase images $(2 \times$ phase-amplified), respectively, at $0 \mathrm{~V}$ (a), $2.0 \mathrm{~V}$ (b), 4.0 $\mathrm{V}(\mathrm{c}), 6.0 \mathrm{~V}(\mathrm{~d}), 8.0 \mathrm{~V}(\mathrm{e})$ and $10.0 \mathrm{~V}$ (f). It can be seen from these images that the phase shift across the p-n junction increases with increasing bias voltage in the thicker part of the film, which indicates that the argon ion milling effectively reduces the surface damage. 
In summary, the combined electron holography-FIB milling was used to observe the electrostatic potential in a biased GaP diode. The FIB milling induces a low resistivity surface damage layer in the specimen; it is therefore essential to remove the damage layer in our experiment [5].

[1] W. D. Rau et al., Phys. Rev. Lett. 82 (1999) 2614.

[2] Z. Wang et al., Appl. Phys. Lett. 80 (2002) 246.

[3] A. C. Twitchett et al., Phys. Rev. Lett. 88 (2002) 238302-1.

[4] K. Sasaki et al., Electron Microscopy 35 (Suppl. 1) (2000) 190.

[5] This work was performed as part of the Active Nano-Characterization and Technology Project, using Special Cooperation Funds from the Ministry of Education, Culture, Sports, Science and Technology of the Japanese government.

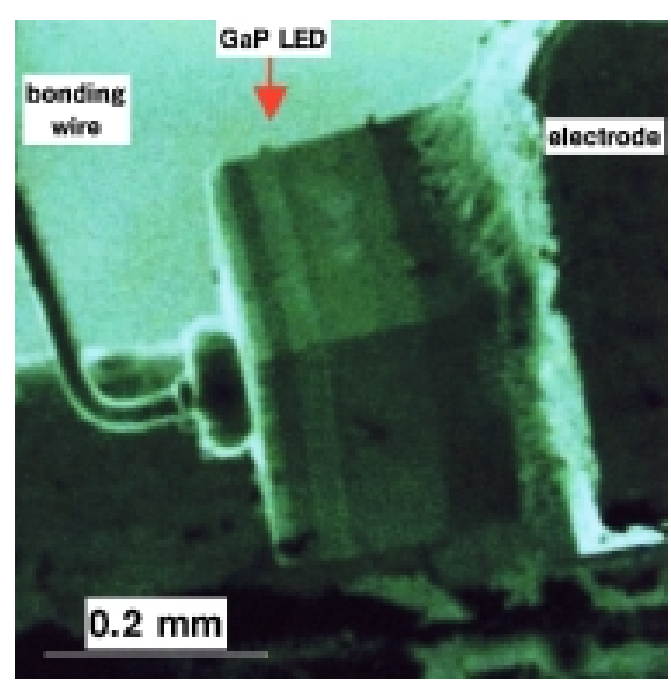

FIG. 1 SIM image of a GaP LED

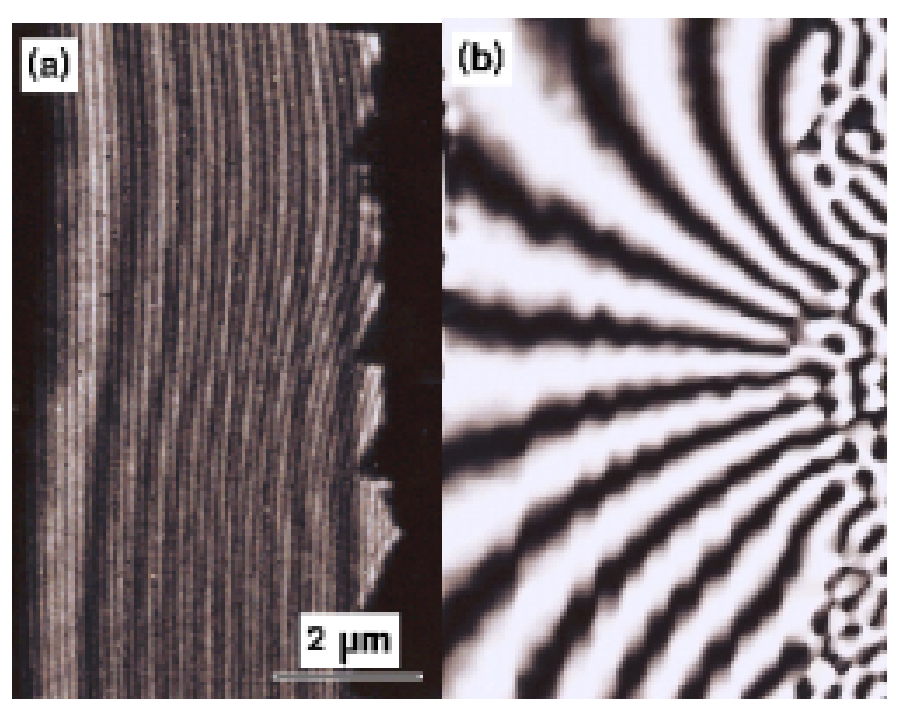

FIG. 2 Electric field around a biased p-n junction

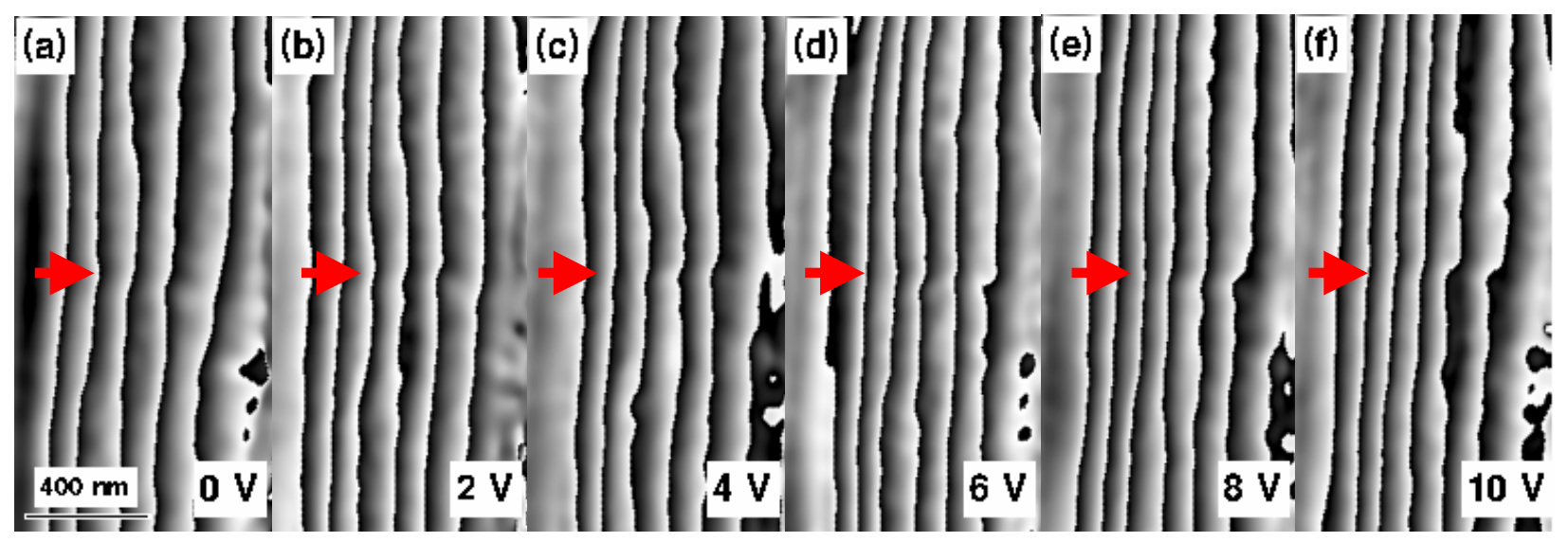

FIG. 3 Reconstructed phase images $(2 \times)$ of a GaP p-n junction at different reverse bias voltages 